\title{
Local In Situ Temperature Measurements from Aluminum Nanoparticles
}

Benjamin K. Miller ${ }^{1}$, H. Hugo Perez Garza ${ }^{2}$, and Matthew Mecklenburg ${ }^{3}$

${ }^{1 .}$ Gatan Inc. 5794 W. Las Positas Blvd. Pleasanton, USA.

2. DENSsolutions B.V., Delft, The Netherlands.

${ }^{3 .}$ Center for Electron Microscopy and Microanalysis, University of Southern California, Los Angeles, USA.

Electron energy loss spectroscopy (EELS) has been used to study the changes in materials since the early days of in situ TEM [1]. Mapping electron energy loss spectra can determine the redistribution of elements in a material's nanostructure, monitor oxidation or reduction, or even observe the exchange of elements from the gaseous or liquid environment into solid structures during growth processes. EELS can also be used to measure the conditions surrounding the sample, including gaseous or liquid environments [1] as well as temperature [2,3]. This is useful, since it can be challenging to measure local conditions inside the TEM using more traditional measurement techniques.

In the present work, we show how EELS can be used to measure local temperature using $\mathrm{Al}$ nanoparticles. The heating holder was a DENSsolutions Wildfire System, which can heat samples to $1300{ }^{\circ} \mathrm{C}$ with low thermal drift [4]. Images of the microheater in this holder and the nanoparticles deposited on the electron transparent window are shown in Figure 1A and 1B, respectively. This holder was controlled using Gatan's Digital Micrograph (or GMS) software. The electron energy loss spectra, like that shown in Figure 1C, were captured using a Gatan K2 Quantum GIF (on a JEOL F200 TEM), which captures counted EELS spectra at rates up to $400 \mathrm{fps}$.

Measurement of local temperature using EELS is possible because the plasmon peak position is dependent on electron density, and this density is dependent on the thermal expansion of the material. We used plasmon energy expansion thermometry (PEET) to determine the change in temperature from the change in plasmon energy [2]. Aluminum nanoparticles are ideal for this measurement, as they have a sharp plasmon peak around $15 \mathrm{eV}$.

The ability to measure temperature locally with EELS leads to several possible experiments. First, the accuracy of the vendor-provided temperature calibration of in-situ holders can be determined, as shown in Figure 2. This is important for in-situ experiments in which accurate measurement of temperature is a necessity. By verifying the temperature accuracy under various conditions, a basis for trusting the nominal temperature given by the holder is established. Second, it becomes possible for researchers to map the spatial distribution of temperature at steady state across the holder. Small regions can be mapped using spectrum imaging, while larger regions can be mapped out manually by acquiring spectra from different areas. A third possibility is to measure temperature under non-equilibrium conditions, monitoring the response of the local temperature to rapid changes in the set temperature with temporal resolution on the order of milliseconds. Data and discussion on all three of these will be presented [5].

References:

[1] PA Crozier, BK Miller, in "Controlled Atmosphere Transmission Electron Microscopy", ed. TW Hansen, JB Wagner, (Springer International Publishing) pp. 95-141. 
[2] M Mecklenburg et al, Science 347 (2015), p. 629-632.

[3] M Mecklenburg, B Zutter, and BC Regan, Phys. Rev. Applied 9 (2018), p 014005.

[4] HHP Garza, et al, 19th International Conference On Solid-State Sensors, Actuators and

Microsystems, (2017), p. 2155-2158.

[5] The authors acknowledge the support from the Center for Electron Microscopy and Microanalysis at the University of Southern California.
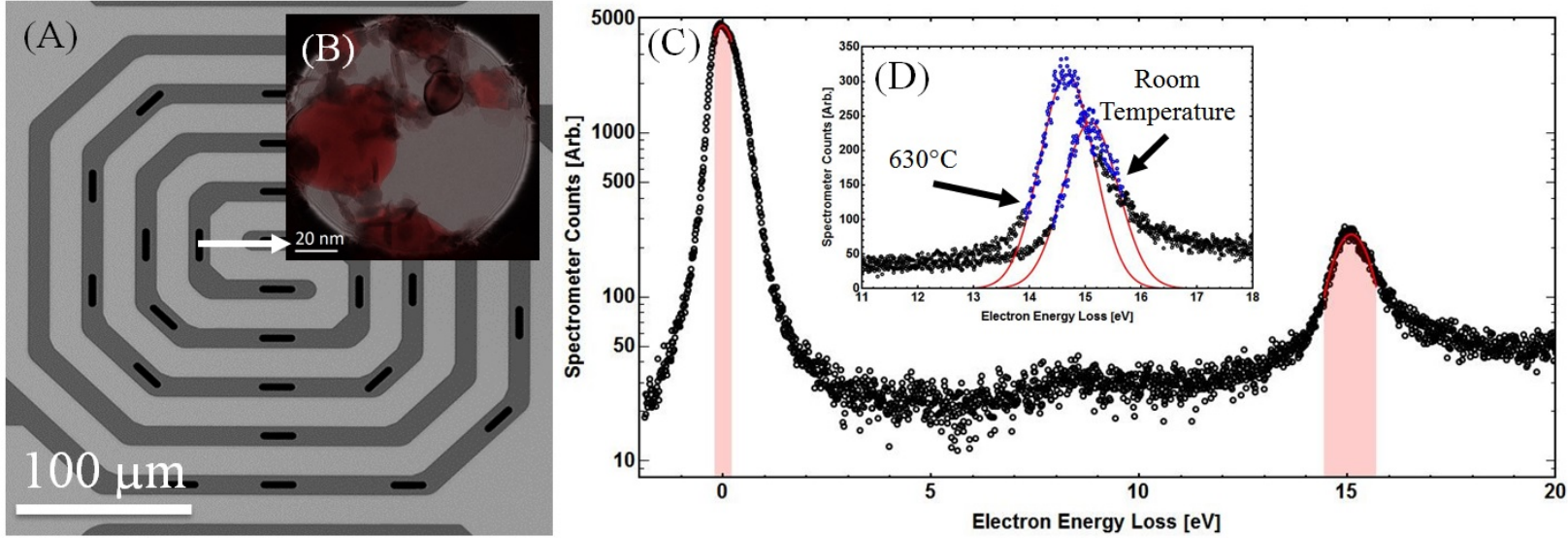

Figure 1. (A) An SEM image of the DENS heater is shown. Each of the electron transparent windows is $100 \mu \mathrm{m}^{2}$ in area and one of the four central windows was used in this heating experiment. Aluminum nanoparticles were deposited on the surface sparsely covering each window. (B) Nanoparticles, shown imaged via EFTEM in red (the circle is defined by the spectrometer, not the chip), sit on top of a $<20 \mathrm{~nm}$ thick silicon nitride membrane covering each hole. (C) Shown is an acquired EELS spectrum at room temperature. The fit regions for the ZLP and the first plasmon peak are shown (the difference in peak position determines the plasmon energy). (D) Fits at room temperature and $630^{\circ} \mathrm{C}$ are shown, as is the dynamic window (centered around the plasmon peak) used to specify the fit region around each peak.
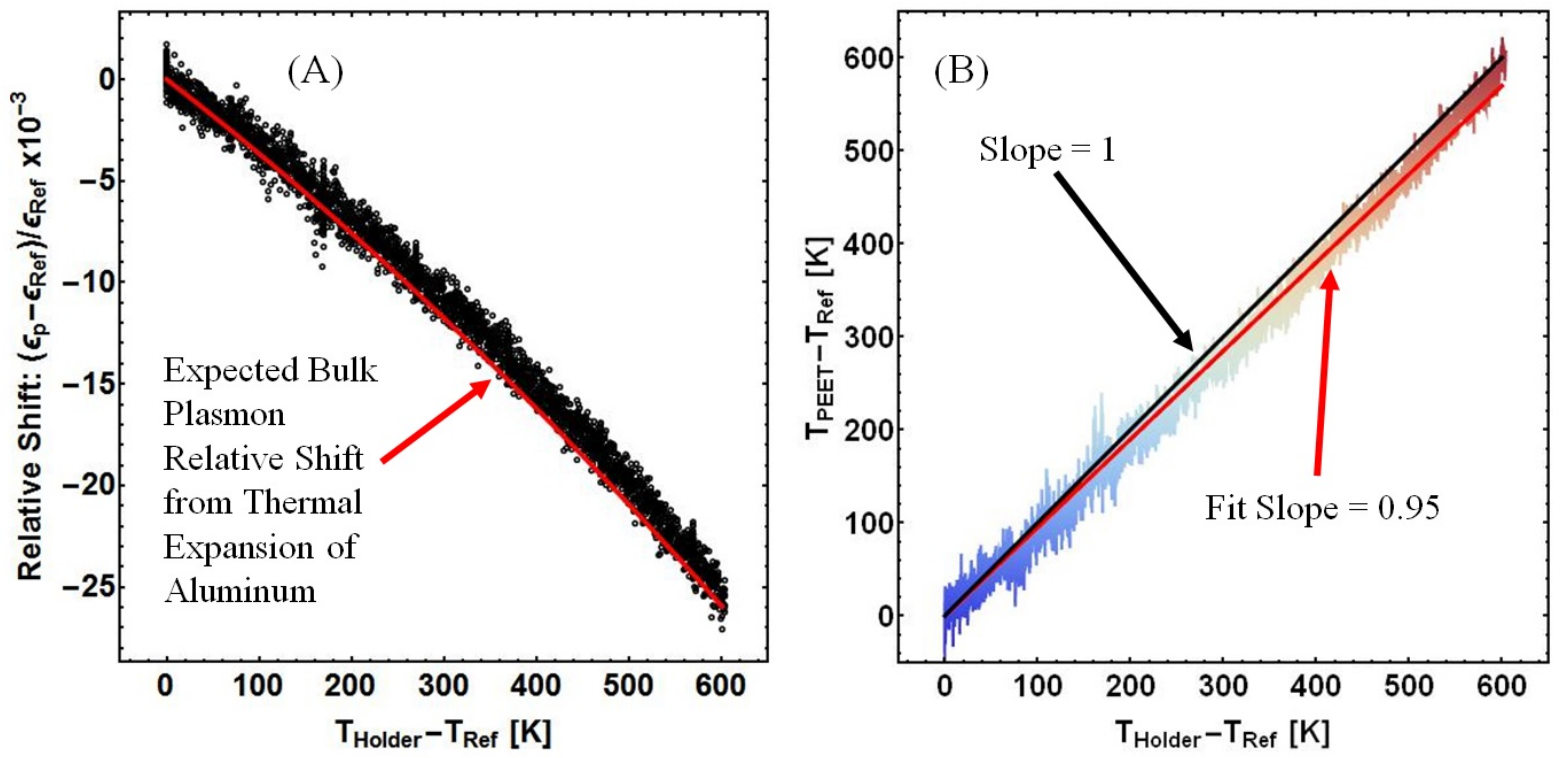

Figure 2. (A) A plot of the relative bulk plasmon shift is shown $\left(T_{\text {Ref }}=\right.$ Room Temperature). The red curve indicates the expected relative shift based on the temperature dependent thermal expansion coefficient in aluminum. (B) The measured shift in temperature from the heating holder is plotted against the corresponding change determined by PEET. The fit slope is determined to be 0.95 , which is close to the expected unity slope. 